\title{
Software Framework for an Ocular Biometric System
}

\author{
Corey D. Holland and Oleg V. Komogortsev \\ Texas State University - San Marcos \\ 601 University Drive, San Marcos TX 78666 \\ ch1570@txstate.edu, ok11@txstate.edu
}

\begin{abstract}
This document describes the software framework of an ocular biometric system. The framework encompasses several interconnected components that allow an end-user to perform biometric enrollment, verification, and identification with most

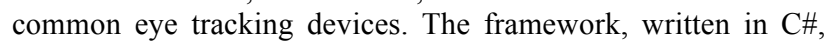
includes multiple state-of-the-art biometric algorithms and information fusion techniques, and can be easily extended to utilize new biometric techniques and eye tracking devices.
\end{abstract}

CR Categories: D.2.13 [Software Engineering] Reusable Software - Reusable libraries; I.5.4 [Pattern Recognition] Applications - Computer vision; K.6.5 [Management of Computing and Information Systems] Security and Protection - Authentication

Keywords: biometrics, eye movements, security, software

\section{Introduction}

Eye movement biometrics is a relatively new sub-field of the biometric discipline, the foundations of which were laid in the 1970s [Noton and Stark 1971], but whose initial investigations did not begin in earnest until the early 2000s [Kasprowski and Ober 2004]. Having existed for less than a decade, eye movement biometrics possess a number of qualities that make them useful and interesting, despite their infancy.

First, due to the unique physical properties of the oculomotor plant and neurological properties of the brainstem control, it is practically infeasible to accurately reproduce human eye movements outside of a living subject, providing an inherent level of counterfeit-resistance and liveness detection [Komogortsev and Karpov 2013]. Second, eye movement biometrics have been shown to enhance the accuracy and counterfeit-resistance of multi-biometric systems [Komogortsev, et al. 2012]. Finally, eye movements can be recorded and processed in real-time using an unmodified camera [Agustin, et al. 2009], thereby allowing the incorporation of eye movement biometrics into existing iris-, retina-, and face-based systems without the need for additional sensors.

In this paper, we describe a software framework for eye movement biometrics that can be easily extended to other biometric modalities.

\section{System Structure}

The ocular biometric system is comprised of three major components, described in detail in the following sections: the user interface, the device wrappers, and the biometric framework. The user interface describes all forward-facing components with which the end-user may interact, the device wrappers provide a

Permission to make digital or hard copies of part or all of this work for personal or classroom use is granted without fee provided that copies are not made or distributed for commercial advantage and that copies bear this notice and the full citation on the first page. Copyrights for third-party components of this work must be honored. For all other uses, contact the Owner/Author.

ETRA 2014, March 26 - 28, 2014, Safety Harbor, Florida, USA.

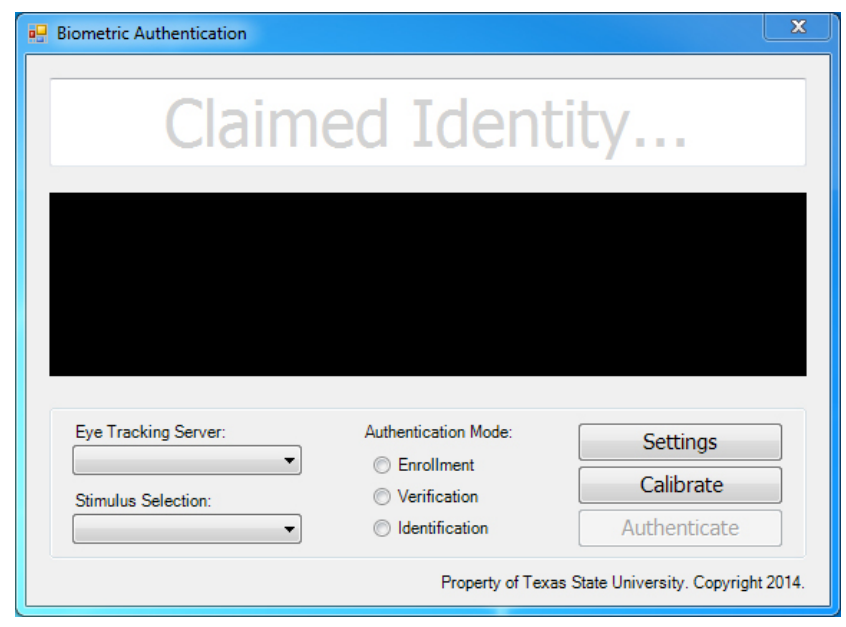

Figure 1. Ocular biometric system user interface.

common interface for various eye tracking devices, and the biometric framework contains the algorithms and procedures necessary to perform biometric authentication.

While the software framework described in this paper focuses primarily on eye movement biometrics, we refer to an ocular biometric system, as the framework can be easily extended to iris-pattern biometrics. In fact, many eye tracking systems provide facilities for iris/retina capture, and modern videooculography techniques allow eye tracking on any device capable of video capture at 15-30 frames per second [Holland and Komogortsev 2012].

\section{User Interface}

The initial window, shown in Figure 1, presents the primary authentication form, through which the end-user provides a claimed identity and selects an available eye tracking device, stimulus, and biometric modality. The authentication form provides feedback through the large status message displayed below the claimed identity textbox.

The settings window allows the user to modify the parameters of the biometric framework, this includes: eye movement classification algorithm and thresholds, interpolation algorithm for missing data points, biometric algorithms, and information fusion algorithms.

The calibration window displays a sequential grid of stimuli, calculating the difference between the measured and predicted gaze points at each stimulus location. This procedure is necessary for most eye tracking devices to allow the system to compensate for known error. 
The stimulus window is generated at run-time from XML files that store the sequence and duration of stimuli. This makes it easy to add and modify the stimuli used for biometric authentication, without the need to modify and compile source code. Further, this allows the end-user to create their own stimuli, making it more difficult to prepare targeted spoof recordings, and increasing the overall flexibility and security of the system.

In its current implementation, the ocular biometric system provides five stimulus routines for biometric authentication. The horizontal stimulus is designed to evoke fixed-amplitude horizontal saccades that span the width of the screen, the vertical stimulus is designed to evoke fixed-amplitude vertical saccades that span the height of the screen, the random stimulus is designed to evoke uniformly random oblique saccades, the reading stimulus is designed to evoke reading saccades for an excerpt of difficult English prose, and the Rorschach stimulus is designed to evoke sub-conscious visual search behavior.

\section{Device Wrappers}

Despite the fact that most commercial eye tracking systems perform similar actions, and provide similar output, the hardware and software interfaces of these devices vary widely. To reduce this variability, we define a common structure for gaze points (a tuple containing the timestamp, $\mathrm{x}-$, and $\mathrm{y}$-coordinates), and an interface that must provide certain basic functions for: connecting to and disconnecting from the device, performing calibration, and retrieving gaze data.

A common eye tracking class uses reflection to identify device wrappers and list all available eye tracking devices at run-time. The end-user selects one of the available devices, and the common class uses the interface to invoke the necessary functions from the device wrapper. While this means that new device wrappers must still be incorporated at compile time, it is as simple as providing a class that maps the relevant API calls to the common interface, and does not involve modification of any existing code.

The ocular biometric system provides device wrappers for Tobii eye tracking systems, though there are plans to include videooculography algorithms that would allow for the easy use of common web cameras and iris/retina devices in the near future.

\section{Biometric Framework}

The biometric framework provides the primary logic for three biometric scenarios: enrollment, verification, and identification. Biometric enrollment is used to submit valid/authentic users to the biometric database. The most recent recording is retrieved from the connected eye tracking device and stored in the biometric database. The biometric database is encrypted and compressed to improve security and reduce space requirements.

Biometric verification is used to validate a user as a supplied identity. The most recent recording is retrieved from the connected eye tracking device, and the recording of the supplied identity is extracted from the biometric database. Biometric comparison and information fusion algorithms are applied to the recordings to generate a probability that the current recording matches the supplied identity in the biometric database. An acceptance threshold determines whether the user is genuine or an imposter. The acceptance or rejection of the authentication attempt is returned to the end-user.
Biometric identification is used to determine the identity of a user. The most recent recording is retrieved from the connected eye tracking device, and the entire biometric database is extracted. Biometric comparison and information fusion algorithms are applied against all recordings in the database, generating a set of probabilities that the current recording matches any given identity in the biometric database. The identity of the closest match is returned to the end-user.

The ocular biometric system provides biometric comparison algorithms for the CEM-P [Holland and Komogortsev 2011], CEM-B [Holland and Komogortsev 2013], and COB [Komogortsev and Holland 2013] biometric paradigms, with plans to add support for OPC [Komogortsev, et al. 2010] in the near future.

\section{Conclusion}

The software framework described in this document provides an open-source implementation of multiple state-of-the-art eye movement biometrics and information fusion algorithms. Further, the ocular biometric system is designed to be easy to incorporate, maintain, and extend.

This work is supported in part by NSF CAREER Grant \#CNS1250718 and NSF GRFP Grant \#DGE-11444666, and NIST Grants \#60NANB10D213 and \#60NANB12D234.

\section{References}

D. NOTON AND L. W. STARK. 1971. Scanpaths in Eye Movements during Pattern Perception. Science 171, 308-311.

P. KASPROWSKI AND J. OBER. 2004. Eye Movements in Biometrics. In European Conference on Computer Vision (ECCV), 248-258.

O. V. KomogortseV AND A. KARPOV. 2013. Liveness Detection via Oculomotor Plant Characteristics: Attack of Mechanical Replicas. In IEEE/IAPR International Conference on Biometrics (ICB), 1-8.

O. V. Komogortsev, A. Karpov, C. D. Holland AND H. PROENÇA. 2012. Multimodal Ocular Biometrics Approach: A Feasibility Study. In Fifth International Conference on Biometrics: Theory, Applications and Systems (BTAS), 1-8.

J. S. Agustin, H. SkovsgaARd, J. P. HANSEn AND D. W. HANSEN. 2009. Low-cost Gaze Interaction: Ready to Deliver the Promises. In Conference on Human Factors in Computing (CHI), 4453-4458.

C. D. Holland AND O. V. KomogortSeV. 2012. Eye Tracking on Unmodified Common Tablets: Challenges and Solutions. In Eye Tracking Research \& Applications (ETRA) Symposium, 1-4.

C. D. Holland AND O. V. Komogortsev. 2011. Biometric Identification via Eye Movement Scanpaths in Reading. In International Joint Conference on Biometrics (IJCB), 1-8.

C. D. Holland and O. V. Komogortsev. 2013. Complex Eye Movement Pattern Biometrics: Analyzing Fixations and Saccades. In IAPR International Conference on Biometrics (ICB), 1-8.

O. V. Komogortsev AND C. D. Holland. 2013. Biometric Authentication via Complex Oculomotor Behavior. In International Conference on Biometrics: Theory, Applications and Systems (BTAS), 1-8.

O. V. KomogortseV, U. K. S. Jayarathna, C. R. ARAgOn AND M. MECHEHOUL. 2010. Biometric Identification via an Oculomotor Plant Mathematical Model. In Eye Tracking Research \& Applications (ETRA) Symposium, 57-60. 